\title{
THE MAX-MIN GROUP CONTEST: WEAKEST-LINK (GROUP) ALL-PAY AUCTION
}

\author{
SUBHASISH M. CHOWDHURY, ${ }^{1}$ DONGRYUL LEE, ${ }^{2}$ AND IRYNA TOPOLYAN ${ }^{3}$ \\ ${ }^{1}$ School of Economics, Centre for Behavioural and Experimental Social Science, and Centre for Compe- \\ tition Policy, University of East Anglia, Norwich NR4 7TJ, UK \\ ${ }^{2}$ Department of Economics, Sungshin University, Seoul 136-742, Republic of Korea \\ ${ }^{3}$ Corresponding author; Department of Economics, University of Cincinnati, 2624 Campus Green Dr., \\ Cincinnati, OH 45212, USA; Tel.: 1-765-418-7169; topolyia@ucmail.uc.edu
}

Abstract. We investigate a group all-pay auction in which each group's effort is represented by the minimum among the effort levels exerted by the group members and the prize is a group-specific public good. Examples of such structure include R\&D competitions, sequential production, supply-chain process, negative product or political campaigns, various sporting events, and territorial conflicts. We fully characterize the symmetric equilibria for two groups. There are four types of equilibria: the pure strategy equilibria in which all (active) players exert the same effort, the semi-pure strategy equilibria in which the players in a group play the same pure strategy whereas those in the other group play the same mixed strategy, the non-degenerate mixed strategy equilibria with continuous support, and the non-degenerate mixed strategy equilibria with discontinuous support. We then analyze the case of the general $n$-group. We find the pure strategy equilibria and specify candidates for the mixed strategy equilibria.

Keywords: Group contest; Weakest link; All-pay auction JEL classifications: C72; D70; D72; H41

Date: February 13, 2015. 


\section{Introduction}

“... It's a race

And there ain't no room

For someone in second place."

- "I'm in a Hurry (And Don't Know Why)" by Alabama released in 1992.

There are frequent instances in which groups get engaged with cut-throat competition (as depicted in the lyrics above) and the strength of a group in the competition is represented by the weakest performance among its group members. This is prominent especially in markets. Take, for example, the case of $R \& D$ race among organizations in which the R\&D team within an organization is stratified with complementary units, or the innovation process within the organization is sequential (Abernathy and Rosenbloom, 1968; Kaplan et al., 2003). Another example is the race between two RJVs to set the standard for a new O-ring (Kremer, 1993) type product. In each of the cases, the organization which has the strongest 'weakest-link' in their innovation process wins the $\mathrm{R} \& \mathrm{D}$ race. Combining these concepts, Hansen and Birkinshaw (2007) conclude that the innovation in an organization is only as effective as the strength of the weakest-link in its 'innovation value chain'.

Similar logic applies to the situation in which products compete through their supply chains to sell in a market. Regarding this, Benton and Maloni (2005) note that "...a product is delivered to the end customer via a supply chain... but a supply chain is only as strong as its weakest link." Slone et al. (2007) reiterate, in the same line, the importance of the strength of the weakest-link in competition among supply chains. Inevitably, the product linked with the strongest supply chain captures (most of) the market.

These examples above can be described as group contests. Contests are a family of games in which players exert costly resources such as effort, time, and money in expectation of winning a valuable prize (Konrad, 2009). In the above given situations firms make irreversible investments in their R\&D process or Supply Chains in order to win a patent or to capture a market segment. Modeling a contest involves specifying the probability of winning as a function of efforts put forth by the players. This function is called a Contest Success Function (CSF). One of the most popular CSFs used in the literature is the all-pay auction (Hillman and Samet, 1987; Hillman and Riely, 1989; Baye et al., 
1996) in which the player choosing the highest effort wins with certainty. ${ }^{1}$ The cut-throat competitions described above can be modeled with all-pay auctions. In the case of group all-pay auctions, groups engage in costly confrontations and each group member may make a costly investment (bid) for its group. A function that translates the bids of individual group members into the group bid is called an Impact Function (Wärneryd, 1998).

In the current study we investigate a group all-pay auction with the weakest-link impact function. In other words, the minimum among the bids made by the individual members within a group represents the group bid of that group, the group whose group bid is the maximum among all the groups wins the prize, and all players lose their bids. As the Max bid among the Min bids turns out to be the winner, we call the contest described above as the Max-Min Group Contest. Following the literature, in the continuation we denote the 'resources' or the 'bids' as the 'effort' expended. We make two assumptions which are also standard in the literature and in line with the examples provided. First, the prize has the nature of a group-specific public good in the sense that the prize is shared by all the members of the winning group, even if one or more members in the winning group does not expend effort. Second, we assume complete information, i.e., the game structure and the parameter values are of common knowledge.

The literature on group contest emerges with Katz et al. (1990) who use a perfectly substitute impact function and a logit-type CSF under symmetric valuation of the prize. Baik (2008) extends this with asymmetric valuation. ${ }^{2}$ It is found that players with lowvaluation for the prize free-ride on the highest-valuation group member(s). The analyses of non-linear impact functions are rather recent. Under logit-type CSF, Lee (2012) uses a weakest-link impact function and finds multiple equilibria with no free-riding. Chowdhury et al. (2013), instead, use a best-shot impact function in which the best effort among the group members represents the group effort. They find that it is possible for the highest valuation player to free ride on a lower-valuation group member in equilibrium. Kolmar and Rommeswinkel (2013) use a Constant Elasticity of Substitution impact function ranging from perfect substitute to weakest link and pin down the convergence conditions for equilibria. All these studies consider every group to follow the same impact function, but

\footnotetext{
${ }^{1}$ Two other canonical CSFs that instead consider noise in individual efforts are the logit-type CSF (Tullock, 1980) and the Tournament (Lazear and Rosen, 1981). Please see Konrad (2009) for a survey.

${ }^{2}$ Ursprung (1990), Baik (1993), Riaz et al. (1995), Dijkstra (1998), Münster (2009) and Choi et al. (2014) also study the group contests with logit-type CSF and group-specific public good prize. Nitzan (1991a,b), Lee (1995), Katz and Tokatlidu (1996) and Wärneryd (1998) consider the prize as a private good.
} 
Chowdhury and Topolyan (2013) analyze a group contest in which it is possible for different groups to follow different impact functions and fully characterize related equilibria.

Turning to the group all-pay auction, the focus of this study, Baik et al. (2001) and Topolyan (2014) consider a perfectly substitute impact function and find free-riding equilibria analogous to the logit-type CSF results. Barbieri et al. (2014) consider a best-shot impact function and find conditions under which multiple players in each group may be active.

In this study we aim to make contributions in three focal areas. First, we contribute to the group contest literature by employing the weakest-link impact function and the all-pay auction CSF. Second, we also contribute to the all-pay auction literature by considering a group all-pay auction and show that under this specific (weakest-link) technology, the resultant equilibria are considerably different from the ones existing studies found. Finally, since the prize in this group contest has the nature of a public good within the winning group, the current study is also related to the literature on the provision of public goods under network externalities; and further contributes to a well-established area of research. ${ }^{3}$

As argued above, plenty of field incidences involve this contest. To add to the cases in industrial organization, the system reliability war between two system provider groups also has similar features with this contest. In all cases the weakest part of the product / system portrays its strength while competing with another product or system. Similarly, when rival organizations are involved in negative advertisement about several dimensions of a product, then the product with the weakest feature in a particular dimension eventually loses market. Even outside of market, in the case of negative electoral campaigning on a set of political issues (Skaperdas and Grofman, 1995), the weakest issue determines the strength of a candidate. ${ }^{4}$ Hence, situations in which multi-dimensional negative campaigning is involved can also be modeled as the Max-Min group contest.

Another distinct example of the Max-Min group contest is territorial conflict. It is observed that different computer viruses and bots often get involved into conflict to capture the same computer or program. The 'Computer World' magazine reported in 2010 that "An upstart Trojan horse program has decided to take on its much-larger rival by stealing data and then removing the malicious program from infected computers. Security

\footnotetext{
${ }^{3}$ This includes studies by Hirshleifer (1983), Bergstrom et al. (1986), Harrison and Hirshleifer (1989), Vicary (1990), Conybeare et al. (1994), Sandler and Vicary (2001), Arce (2001), Vicary and Sandler (2002), Varian (2004), Cornes and Hartley (2007), and Lei et al. (2007).

${ }^{4}$ This also relates to the literature on Sabotage. Please see Chowdhury and Gürtler (2014) for a survey.
} 
researchers observe that the relatively unknown Spy Eye toolkit added this functionality just a few days ago in a bid to displace its larger rival, known as Zeus. The feature, called "Kill Zeus," apparently removes the Zeus software from the victims PC, giving Spy Eye exclusive access to usernames and passwords. ${ }^{5}$ " Each virus can be considered as a group of codes. While engaged in territorial conflict, whether a virus will ultimately be the victim of its rival and be abolished depends crucially on the weakest part of its codes. Similar feature can be observed in the nature. National Geographic (2006) observes Turf battle among Borneo Sea Eagles in which a pair of Sea Eagles fight off intruder Eagles who try to catch fish in the territory covered by the pair. ${ }^{6}$ At the time of the fight, once one of the group members leaves the battle, the group loses. Hence, this turns out to be a group conflict with weakest-link technology as well.

The last set of examples come from Sports. Consider, for instance, team pursuit sporting events such as team races in speed skating and female cycling. In these events each team's record is measured as the time when the last member of the team finishes the race and the winning prize goes to the team whose record is the fastest. Also, in both summer and winter Olympic team events based on synchronization, such as synchronized swimming, rhythmic gymnastics, or pairs (figure) skating, the team performance literally depends on the lowest performer of the team. These cases, thus, turn out to be copybook examples of the Max-Min group contest.

All these examples are comprised of diverse background and nature. Nevertheless they all reflect situations in which groups engage in cut-throat competition and the 'strength' of a group depends on the lowest effort exerted by a member of the group. This, however, is not surprising for the cases of computer viruses, Eagles, and negative campaigns due to their attacking nature. When groups get involved in attack games such as these, then the group members are essentially connected with a weakest-link technology, and the survival of a group depends on its weakest group member (Clark and Konrad, 2007; Kovenock and Roberson, 2010). For the other examples, the structure of the contest itself makes them in resemblance with the Max-Min group contest.

Although there are various field examples of this type of group all-pay auction with weakest-link technology, no study to date has tried to analyze this. In the current study we

\footnotetext{
${ }^{5}$ http://www.computerworld.com/s/article/9154618/New_Russian_botnet_tries_to_kill_rival. This, however, is not an exception. Similar incidences noted by, among others, Layden (2008) in Channel Register Magazine. Details are available here: http://www.channelregister.co.uk/2008/02/28/rootkit_wars/.

${ }^{6}$ http://rockyforkfarms.com/eagle-vs-eagle-in-mid-air/
} 
characterize equilibria for the Max-Min group contest and discuss the related implications. Standard all-pay auction literature, be it regarding individual players (Baye et al., 1996) or groups (Baik et al., 2001), shows that pure strategy equilibrium does not exist. However, in our model, we find that a continuum of pure strategy equilibria might co-exist with three characteristically different mixed strategy equilibria. In the pure strategy equilibria, all (active) players expend the same effort. A special type of mixed strategy equilibrium exists in which every member in a group exerts the same effort whereas every member in the other group plays the same mixed strategy. We call this a 'semi-pure strategy' equilibrium. In addition to this semi-pure strategy equilibrium, there are two types of non-degenerate mixed strategy equilibria: the mixed strategy equilibria with continuous support and those with discontinuous support. These results, we believe, contribute to the areas of group contests, all-pay auctions, and public goods with network externalities.

The rest of the paper proceeds as follows. Section 2 constructs a two-group model and characterizes all the possible equilibria. This helps to keep brevity in our analysis, because many of the quantitative results in the two-group model follow through to Section 3 which analyzes a general $n$-group model with different group sizes. In Section 3 , we characterize the pure strategy equilibria and introduce the candidates for the mixed strategy equilibria for the general case. We discuss the results and corresponding implications in Section 4.

\section{The two-group model}

There are two groups, 1 and 2. Group $g$ consists of $k_{g} \geq 2$ risk-neutral players, where $g=1,2$. Denote by $I_{g}$ the index set of all players in group $g$. The prize is a group-specific public good, and the valuation of the prize is the same for every player in group $g$ (denoted by $v_{g}$ ). Assume, without loss of generality, that $v_{1} \geq v_{2}$.

Denote the effort level of player $i$ in group $g$ by $x_{g, i}$, and the vector of effort levels $\left(x_{g, 1}, \cdots, x_{g, k_{g}}\right)$ by $x_{g}$. Given player $i$ in group $g$ and a tuple of effort levels $\left(x_{1}, x_{2}\right)$, denote by $x_{-i}^{g}$ the effort levels of all players other than player $i$ in group $g$, i.e.,

$$
x_{-i}^{g}=\left\{\left(x_{g, 1}, \cdots, x_{g, i-1}, x_{g, i+1}, \cdots x_{g, k_{g}}\right), x_{-g}\right\},
$$

where $x_{-g}$ is the vector of effort levels of the other group.

Definition 2.1. Given a vector of effort levels of the members in group $g, x_{g}$, group $g$ 's effort level is a non-negative real number $B\left(x_{g}\right)$, where $B$ is a (weakest link) function of 
$k_{g}$ variables of the following form:

$$
B\left(x_{g}\right)=\min _{i \in I_{g}} x_{g, i}
$$

The cost function for the effort is the same for all players and $c(x)=x$ for any effort level $x \geq 0$.

We assume both groups compete in an all-pay auction (see Hillman and Riley, 1989; Baye et al., 1996). Let $P_{g}$ denote the probability of group $g$ 's winning, then the contest success function has the following form:

$$
P_{g}\left(x_{g}, x_{-g}\right)=\left\{\begin{array}{lll}
1 & \text { if } & B\left(x_{g}\right)>B\left(x_{-g}\right) \\
\frac{1}{2} & \text { if } & B\left(x_{g}\right)=B\left(x_{-g}\right) \\
0 & \text { if } & B\left(x_{g}\right)<B\left(x_{-g}\right) .
\end{array}\right.
$$

Consequently, the payoff function of player $i$ in group $g$ has the following form:

$$
u_{g, i}\left(x_{g}, x_{-g}\right)=\left\{\begin{array}{rlll}
v_{g}-x_{g, i} & \text { if } & B\left(x_{g}\right)>B\left(x_{-g}\right) \\
\frac{v_{g}}{2}-x_{g, i} & \text { if } & B\left(x_{g}\right)=B\left(x_{-g}\right) \\
-x_{g, i} & \text { if } & B\left(x_{g}\right)<B\left(x_{-g}\right) .
\end{array}\right.
$$

Definition 2.2. A strategy of player $i$ in group $g$ is a probability distribution over a subset of $\mathbb{R}_{+}$(the set of effort levels of player $i$ ), denoted by $s_{g, i}$. Denote the set of all strategies of player $i$ by $\mathbb{S}_{g, i}$.

Definition 2.3. Group $g$ 's strategy is a $k_{g}$-tuple of its members' strategies $\left(s_{g, 1}, \cdots, s_{g, k_{g}}\right)$. Group g's strategy is denoted by $s_{g}$. The set of all strategies of group $g$ is denoted by $\mathbb{S}_{g}$, and the set of all strategies for the game by $\mathbb{S}$.

We extend the payoff function $u$ from the set of pure strategies $\mathbb{R}_{+}^{k_{1}+k_{2}}$ to the set of mixed strategies $\mathbb{S}$ as follows. Fix a strategy $s \in \mathbb{S}$; let $F_{g}, F_{g, i}$, and $F_{-g}$ be the probability measures on $\mathbb{R}_{+}$induced by $s_{g}, s_{g, i}$, and $s_{-g}$, respectively. Note that, because each group's effort is determined by the weakest-link fashion, $F_{g}=1-\prod_{i \in I_{g}}\left(1-F_{g, i}\right)$. Intuitively, $F_{g}$ represents the probability distribution induced on $\mathbb{R}_{+}$by $s_{g}$. Then the payoff of player $i$ 
in group $g$ is computed as follows:

$$
u_{g, i}(s)=\int_{\mathbb{R}_{+}} v_{g} F_{-g}(x) d F_{g}(x)-\int_{\mathbb{R}_{+}} x d F_{g, i}(x) .
$$

The first integral in Equation 2.4 represents the expected value of winning the prize, and the second integral represents the expected cost of effort for player $i$.

Definition 2.4. Given a strategy $s_{g, i}$ of player $i$ in group $g$, the individual support $S_{g, i}$ is the closure of set of all points of increase of the cumulative distribution function $F_{g, i}$ corresponding to $s_{g, i}$ :

$$
S_{g, i}=C l\left(\left\{x \in \mathbb{R}_{+}: F_{g, i}(x-\epsilon)<F_{g, i}(x)<F_{g, i}(x+\epsilon) \text { for all } \epsilon>0\right\}\right) .
$$

Definition 2.5. Similarly, given group g's strategy $s_{g}$, the group support of $s_{g}$ is the closure of set of all points of increase of the cumulative distribution function $F_{g}$ corresponding to $s_{g}$, and will denoted by $S_{g}$ :

$$
S_{g}=C l\left(\left\{x \in \mathbb{R}_{+}: F_{g}(x-\epsilon)<F_{g}(x)<F_{g}(x+\epsilon) \text { for all } \epsilon>0\right\}\right) .
$$

Note that a support may fail to be connected. This happens when a subset of the convex hull of the support is omitted by a player, i.e., chosen with probability zero.

Definition 2.6. A tuple $x^{\star}=\left(x_{1}^{\star}, x_{2}^{\star}\right)$, where $x_{g}^{\star}$ is group g's effort level (see Definition 2.1), is called a pure strategy equilibrium if

$$
u_{g, i}\left(x_{g, i}^{\star}, x_{-i}^{g \star}\right) \geq u_{g, i}\left(x_{g, i}, x_{-i}^{g \star}\right) \text { for every player } i \text { in group } g \text { and every } x_{g, i} \in \mathbb{R}_{+} \text {. }
$$

Definition 2.7. A tuple $s^{\star}=\left(s_{1}^{\star}, s_{2}^{\star}\right)$, where $s_{g}^{*}$ is group g's strategy (see Definition 2.3), is called a mixed strategy equilibrium if

$$
u_{g, i}\left(s_{g, i}^{\star}, s_{-i}^{g \star}\right) \geq u_{g, i}\left(s_{g, i}, s_{-i}^{g \star}\right) \text { for every player } i \text { in group } g \text { and every } s_{g, i} \in \mathbb{S}_{g, i}{ }^{7}
$$

Note that a pure strategy equilibrium is a special case of a mixed strategy equilibrium, but we specifically define it here, because there is generally no pure strategy equilibrium in all-pay auctions. The following result is obvious and we state it without a formal proof.

\footnotetext{
${ }^{7}$ The term $s_{-i}^{g \star}$ is defined analogously to $x_{-i}^{g \star}$.
} 
Theorem 2.8. All equilibria in pure strategies are as follows.

$$
x_{g, i}=\lambda \quad \text { for all } \quad i \in I_{g}, g=1,2
$$

where $\lambda \in\left[0, \frac{v_{2}}{2}\right]$. Furthermore the total effort expended in the pure strategy equilibria is $\left(k_{1}+k_{2}\right) \lambda$, which varies between 0 and $\frac{\left(k_{1}+k_{2}\right) v_{2}}{2}$.

Hence, even under an all-pay auction CSF, there exist pure strategy equilibria. This is the opposite of what is observed in standard all-pay auctions when only mixed strategy equilibria exist.The intuition behind this is simple. Due to the weakest-link effort technology, players use symmetric strategies within each group resulting in the PSNE.

Now we discuss the non-degenerate mixed strategy equilibria in this model. We focus on symmetric equilibria in which the players within a group employ the same strategy. For the purpose of brevity, from now on we use the term mixed strategy equilibria instead of non-degenerate symmetric mixed strategy equilibria. First, let us investigate so-called semi-pure strategy equilibria in which some players employ a pure strategy, while others use a mixed strategy.

Definition 2.9. Given an individual support $S_{g, i}$ of player $i$, the upper bound of the support is

$$
\bar{s}_{g, i}=\sup \left\{x: x \in S_{g, i}\right\} .
$$

Similarly, the lower bound of the support is

$$
\underline{s}_{g, i}=\inf \left\{x: x \in S_{g, i}\right\} .
$$

The upper and lower bounds of a group support $S_{g}$ are defined analogously, with $S_{g}$ in place of $S_{g, i}$ in the above definition. For each group $g$, let $S_{g+}^{\star}=S_{g}^{\star} \cap \mathbb{R}_{++}=\{x>0$ : $\left.x \in S_{g}^{\star}\right\}$. Let $\underline{s}_{g+}^{\star}=\inf \left\{x: x \in S_{g+}^{\star}\right\}$ and $\bar{s}_{g+}^{\star}=\sup \left\{x: x \in S_{g+}^{\star}\right\}$.

In the Appendix, we present all the proofs and the auxiliary results, Lemmata 4.1, 4.2, 4.3, and Corollary 4.4, which describe the properties of the mixed strategy equilibria. The following theorem characterizes the semi-pure strategy equilibria.

Theorem 2.10. The symmetric semi-pure strategy equilibria are as follows. 
(1) Each player in group 1 contributes $\frac{v_{2}}{2}(1-a)^{k_{2}-1}$ and each player in group 2 contributes zero with probability $a$ and $\frac{v_{2}}{2}(1-a)^{k_{2}-1}$ with probability $1-a$, for $0<a<1 .^{8}$

(2) If $v_{1}=v_{2}=v$, then in addition there exists a continuum of equilibria where, for $0<a<1$, each player in group 1 contributes 0 with probability a and $\frac{v}{2}(1-a)^{k_{1}-1}$ with probability $1-a$ and each player in group 2 contributes $\frac{v}{2}(1-a)^{k_{1}-1}$.

This is another type of equilibrium which is not very common for all-pay auction. This type of equilibrium is obtained again due to the specific network externalities generated by the weakest-link technology.

Note that it is not an equilibrium for each player in one group to randomize over two strictly positive effort levels, while all players of the other group expend some positive effort level with probability one. This is because any player of the former group would deviate from the lower bound of their group support to zero. Notice that supports of the type $\left[s^{1}, s^{2}\right] \cup\left[s^{3}, s^{4}\right]$, that is, piecewise-continuous supports, are not possible for the following reason. By Lemma 4.2 in the Appendix, no player puts mass at either $s^{2}$ or $s^{3}$. Therefore for a sufficiently small $\epsilon>0$, efforts in $\left(s^{3}, s^{3}+\epsilon\right)$ yield only a small increase in the group's chances of winning, compared to effort level $s^{2}$, while $s^{2}$ provides a discrete reduction in costs compared to $\left(s^{3}, s^{3}+\epsilon\right)$, thus any player would shift mass from $\left(s^{3}, s^{3}+\epsilon\right)$ to $s^{2}$, leading to a contradiction. Similar considerations show that it is not possible to sustain a strictly positive $\underline{s}_{+}$in any semi-symmetric mixed strategy equilibrium.

From Lemmata 4.2, 4.3 and Corollary 4.4, we see that if the equilibrium supports are nontrivial (i.e., non-singletons), then the following are the only possible candidates for the mixed strategy equilibria. In equilibrium, the support of each player $i$ in group $g$ should be either:

(1) the interval $\left[0, \bar{s}^{\star}\right]$;

(2) the union $\left[0, \tilde{s}^{\star}\right] \cup \bar{s}^{\star}$.

More specifically, the following results characterize all (symmetric) mixed strategy equilibria. Let every player in group 1 randomize according to a $\operatorname{cdf} F$; denote the corresponding cdf for a player in group 2 by $G$.

\footnotetext{
${ }^{8}$ Note that, when $a=0$ and $a=1$, the continuum of the semi-pure strategy equilibria degenerate to the pure strategy equilibria where each player contributes $\frac{v_{2}}{2}$ or 0 , respectively.
} 
Theorem 2.11. A mixed strategy equilibrium where all players randomize over some interval without a gap is unique.

(1) If $\frac{k_{1} v_{2}}{k_{2} v_{1}} \leq 1$, then the support is $S_{g, i}^{\star}=\left[0, \bar{s}^{\star}\right]$ for all players, where $\bar{s}^{\star}=\left(\frac{k_{1} v_{2}}{k_{2} v_{1}}\right)^{k_{2}}$. $\frac{k_{2} v_{1}}{k_{1}+k_{2}-1}$,

$$
\begin{gathered}
F(x)=1-\left(1-\frac{x}{\bar{s}^{\star}}\right)^{\frac{1}{k_{1}+k_{2}-1}}, \text { and } \\
G(x)=\left(1-\frac{k_{1} v_{2}}{k_{2} v_{1}}\right)+\frac{k_{1} v_{2}}{k_{2} v_{1}} F(x)=1-\frac{k_{1} v_{2}}{k_{2} v_{1}}\left(1-\frac{x}{\bar{s}^{\star}}\right)^{\frac{1}{k_{1}+k_{2}-1}} .
\end{gathered}
$$

(2) If $\frac{k_{1} v_{2}}{k_{2} v_{1}}>1$, then the support is $S_{g, i}^{\star}=\left[0, \bar{s}^{\star}\right]$ for all players, where $\bar{s}^{\star}=\left(\frac{k_{2} v_{1}}{k_{1} v_{2}}\right)^{k_{1}}$. $\frac{k_{1} v_{2}}{k_{1}+k_{2}-1}$,

$$
\begin{gathered}
G(x)=1-\left(1-\frac{x}{\bar{s}^{\star}}\right)^{\frac{1}{k_{1}+k_{2}-1}} \\
F(x)=\left(1-\frac{k_{2} v_{1}}{k_{1} v_{2}}\right)+\frac{k_{2} v_{1}}{k_{1} v_{2}} G(x)=1-\frac{k_{2} v_{1}}{k_{1} v_{2}}\left(1-\frac{x}{\bar{s}^{\star}}\right)^{\frac{1}{k_{1}+k_{2}-1}} .
\end{gathered}
$$

Corollary 2.12. (Symmetric valuations) Assume $v_{1}=v_{2}=v$ and without loss of generality $k_{1} \leq k_{2}$, then the unique mixed strategy equilibrium where all players randomize over some interval without a gap is as follows. The support is $S_{g, i}^{\star}=\left[0, \bar{s}^{\star}\right]$ for all players, where $\bar{s}^{\star}=\left(\frac{k_{1}}{k_{2}}\right)^{k_{2}} \cdot \frac{k_{2} v}{k_{1}+k_{2}-1}$,

$$
\begin{gathered}
F(x)=1-\left(1-\frac{x}{\bar{s}^{\star}}\right)^{\frac{1}{k_{1}+k_{2}-1}}, \text { and } \\
G(x)=\left(1-\frac{k_{1}}{k_{2}}\right)+\frac{k_{1}}{k_{2}} F(x)=1-\frac{k_{1}}{k_{2}}\left(1-\frac{x}{\bar{s}^{\star}}\right)^{\frac{1}{k_{1}+k_{2}-1}} .
\end{gathered}
$$

Corollary 2.13. Let $s^{\star}$ be the mixed strategy equilibrium of Theorem 2.11. If $\frac{k_{1} v_{2}}{k_{2} v_{1}} \leq 1$, then every player in group 1 earns equilibrium payoff $u_{1}\left(s^{\star}\right)=1-\frac{k_{1} v_{2}}{k_{2} v_{1}}$, and every player in group 2 earns $u_{2}\left(s^{\star}\right)=0$. If $\frac{k_{1} v_{2}}{k_{2} v_{1}}>1$, then $u_{1}\left(s^{\star}\right)=0$ and $u_{2}\left(s^{\star}\right)=1-\frac{k_{2} v_{1}}{k_{1} v_{2}}$.

Given a function $f: \mathbb{R}_{+} \rightarrow \mathbb{R}_{+}$, let $J_{f}(x)$ denote the jump of $f$ at $x \in \mathbb{R}$. In other words, $J_{f}(x)$ is the mass that $f$ puts at $x$ (recall that if $f$ is continuous at $x$, then $J_{f}(x)=0$ ).

Define $\tilde{s}$ as the upper bound of the continuous part of the support. The next theorem pins down the equilibria with possible disjoint support. 
Theorem 2.14. Equilibria where the support of each player is of the form $\left[0, \tilde{s}^{\star}\right] \cup \bar{s}^{\star}$ are as follows:

(1) If $k_{1}=k_{2}$, then $G(x)=1-\frac{v_{2}}{v_{1}}+\frac{k_{1} v_{2}}{k_{2} v_{1}} F(x), F(0)=0$, and $F$ is continuous for all $0 \leq x \leq \tilde{s}_{\dagger} ; J_{F}\left(\bar{s}_{\dagger}\right)=a$, and $J_{G}\left(\bar{s}_{\dagger}\right)=a \frac{v_{2}}{v_{1}}$, where $0<a<1$.

(2) (a) If $k_{1}>k_{2}$, then $G(x)=1-\frac{v_{2}}{v_{1}}+\frac{k_{1} v_{2}}{k_{2} v_{1}} F(x), F(0)=0$, and $F$ is continuous for all $0 \leq x \leq \tilde{s}_{\ddagger} ; J_{F}\left(\bar{s}_{\ddagger}\right)=a$, and $J_{G}\left(\bar{s}_{\ddagger}\right)=a \frac{v_{2}}{v_{1}}$, where $\max \left\{0, \frac{k_{1} v_{2}-k_{2} v_{1}}{v_{2}\left(k_{1}-k_{2}\right)}\right\} \leq$ $a<1$.

(b) If $\frac{k_{1} v_{2}}{k_{2} v_{1}}>1$, then, in addition, the following equilibria exist.

$F(x)=\left[1-a_{1}-\frac{k_{2} v_{1}}{k_{1} v_{2}}+\frac{k_{2}}{k_{1}} a_{1}\right]+\frac{k_{2} v_{1}}{k_{1} v_{2}} G(x), G(0)=0$, and $G$ is continuous for all $0 \leq x \leq \tilde{s}_{\diamond} ; J_{F}\left(\bar{s}_{\diamond}\right)=a$, and $J_{G}\left(\bar{s}_{\diamond}\right)=a \frac{v_{2}}{v_{1}}$, where $0<a \leq \frac{k_{1} v_{2}-k_{2} v_{1}}{v_{2}\left(k_{1}-k_{2}\right)}$.

(3) If $k_{1}<k_{2}$, then $G(x)=1-\frac{v_{2}}{v_{1}}+\frac{k_{1} v_{2}}{k_{2} v_{1}} F(x), F(0)=0$, and $F$ is continuous for all $0 \leq x \leq \tilde{s}_{\nabla} ; J_{F}\left(\bar{s}_{\nabla}\right)=a$, and $J_{G}\left(\bar{s}_{\nabla}\right)=a \frac{v_{2}}{v_{1}}$, where $0<a<1$.

Corollary 2.15. Equilibrium payoffs in mixed strategy equilibria of Theorem 2.14 are as follows.

(1) If $k_{1}=k_{2}$, then each player in group 1 earns $u_{1}\left(s^{\star}\right)=1-\frac{v_{2}}{v_{1}}$, and every player in group 2 earns $u_{2}\left(s^{\star}\right)=0$.

(2) If $k_{1}>k_{2}$, then

(a) If $F(0)=0$, then $u_{1}\left(s^{\star}\right)=1-a_{1} \frac{v_{2}}{v_{1}}-\frac{k_{1} v_{2}}{k_{2} v_{1}}\left(1-a_{1}\right)$ and $u_{2}\left(s^{\star}\right)=0$.

If $\frac{k_{1} v_{2}}{k_{2} v_{1}}>1$, then $u_{1}\left(s^{\star}\right)=0$ and $u_{2}\left(s^{\star}\right)=1-a_{1}-\frac{k_{2} v_{1}}{k_{1} v_{2}}+\frac{k_{2}}{k_{1}} a_{1}$.

(3) If $k_{1}<k_{2}$, then $u_{1}\left(s^{\star}\right)=1-a_{1} \frac{v_{2}}{v_{1}}-\frac{k_{1} v_{2}}{k_{2} v_{1}}\left(1-a_{1}\right)$ and $u_{2}\left(s^{\star}\right)=0$.

Theorems 2.8, 2.10, 2.11, and 2.14 characterize all equilibria. Note that the equilibria are definitely not pay-off equivalent. In the equilibria of Theorems 2.11 and Theorem 2.14, at least one group earns zero payoff. In contrast, there is a continuum of pure strategy equilibria where all players earn a positive payoff (Theorem 2.8). Figure 1 helps to visualize the distinction between equilibria of Theorems 2.11 and 2.14 .

[Figure 1 about here] 

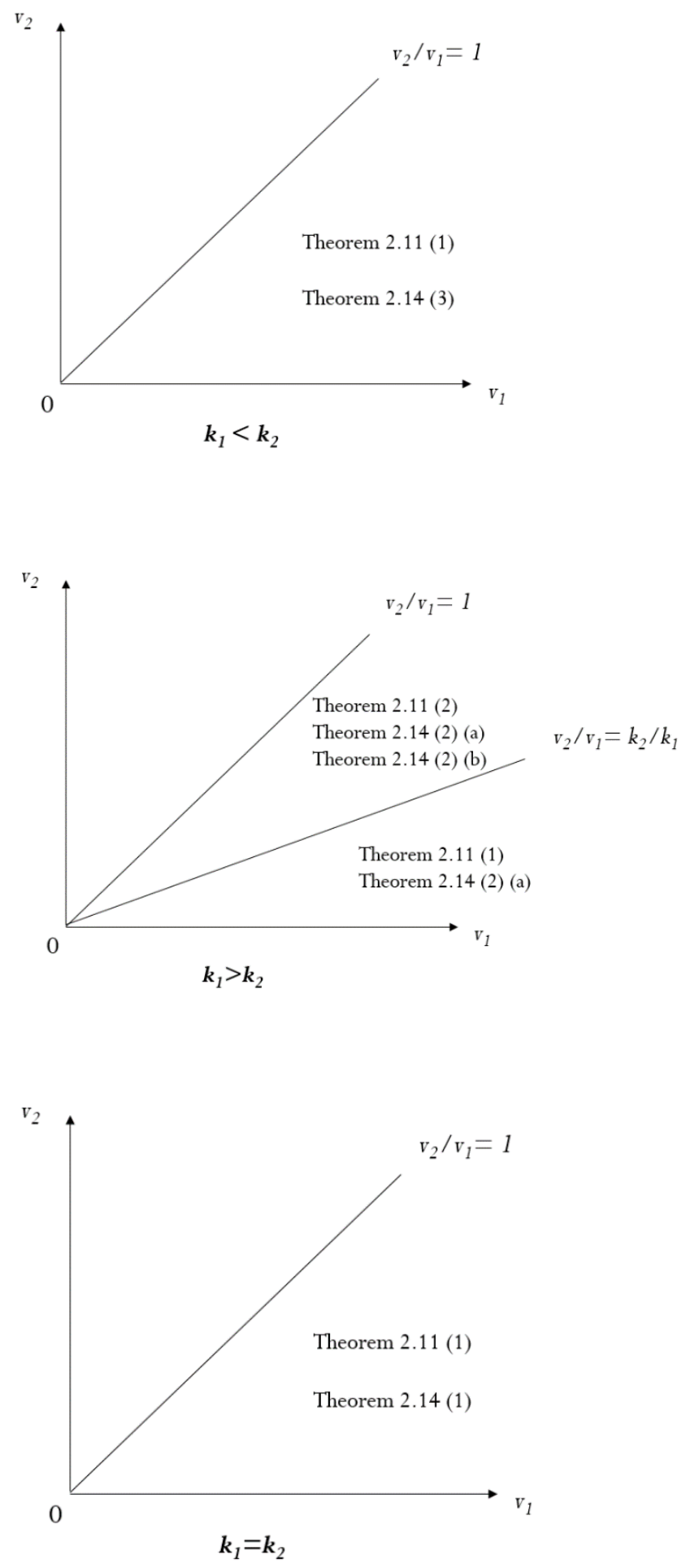

Figure 1. Equilibria in the model 
Although we generally cannot obtain a closed-form solution for $\tilde{s}$ and $\bar{s}$ in Theorem 2.14 , in some simple cases we are able to do so. Consider the following example.

Example 2.16. Suppose two groups compete in an all-pay auction with the weakest-link impact function and a group-specific public good prize. Group 1 has two players, and group 2 has three players. The group valuations are $v_{1}=2$ and $v_{2}=1$. Group 1 is a stylized representation of a small and strong group (in the sense of group valuation). There exists a continuum of pure strategy equilibria where each player expends an effort of $\lambda$, where $0 \leq \lambda \leq \frac{1}{2}$. In the semi-pure equilibrium each player in group 1 always contributes $\frac{1}{8}$, while each player in group 2 contributes zero and $\frac{1}{8}$ with equal probability. Since $\frac{k_{1} v_{2}}{k_{2} v_{1}}=\frac{1}{3}<1$, Theorem 2.11.1 implies that there exists a mixed strategy equilibria of the following form. Each player in group 1 randomizes according to the cdf $F(x)=1-(1-18 x)^{\frac{1}{4}}$, and each player in group $2-G(x)=1-\frac{1}{3}(1-18 x)^{\frac{1}{4}}$, over the common support $\left[0, \frac{1}{18}\right]$. Each player earns in group 1 an equilibrium payoff of $\frac{2}{3}$, while each player in group 2 earns zero. As expected, the larger group is disadvantaged and earns nothing, while the "small and strong" group enjoys a positive payoff.

Moreover, Theorem 2.14.3 implies that there exists a continuum of mixed strategy equilibria with piecewise-continuous supports possessing atoms, as follows. Each player in group 1 randomizes according to the cdf $F$ with $F(0)=0$, which is continuous for all $0 \leq x \leq \tilde{s}$ and puts mass $0<a<1$ at the upper bound of the continuous part of the support. Each player in group 2 randomizes according to the $\operatorname{cdf} G(x)=\frac{1}{2}+\frac{1}{3} F(x)$ over $[0, \tilde{s}]$ and puts mass $\frac{a}{2}$ at $\bar{s}$. We compute the parameters of the distributions of the players' strategies as follows. Equation 4.20, which determines $F$, becomes

$$
\frac{1}{18}(2+a-2 F(x))^{2}(1-F(x)) d F=d x
$$

Integrating both sides of (2.6) and using the condition $F(0)=0$, we obtain the following implicit function which defines $F$ as a function of $x$.

$$
(2+a)^{2} F(x)-\frac{1}{2}(2+a)(6+a) F(x)^{2}+\frac{4}{3}(3+a) F(x)^{3}-F(x)^{4}=18 x
$$


Observe that since $0<a<1$ and $0<F(x)<1-a$ for all $0<x<\tilde{s}$, the expression $(2+a-2 F(x))^{2}(1-F(x))$ is always nonzero. Therefore by the Implicit Function Theorem there exists a unique continuously differentiable function $F(x)$ satisfying Equation 2.7. Using the condition $F(\tilde{s})=1-a$ we can calculate $\tilde{x}$ from Equation 2.7.

$$
\left.\tilde{s}=\frac{1}{108}\left(-17 a^{4}+3 a^{2}+8 a+6\right)\right)
$$

Finally, we can calculate the isolated point where each player puts mass, $\bar{s}$, from Equation 4.15 .

$$
\left.\bar{s}=\frac{1}{216}\left(-7 a^{4}+6 a^{2}+16 a+12\right)\right)
$$

Notice that when $a=0$, such equilibrium degenerates to the mixed strategy equilibrium of Theorem 2.11.1 with the upper bound of the support equals $\frac{1}{18}$; when $a=1$, the equilibrium degenerates to the semi-pure equilibrium. The plot of $\tilde{s}$ and $\bar{s}$ for each value of $a \in[0,1]$ is presented in Figure 2. The equilibrium cdfs are presented in Figure 3. The top part of the figure corresponds to the mixed strategy equilibrium where group 1 puts no mass at the upper bound of the continuous part of the support. The bottom part of Figure 3 corresponds to the semi-pure equilibrium.

[Figure 2 about here]

[Figure 3 about here]

Each player in group 1 receives a payoff of $u_{1}=\frac{2}{3}-\frac{1}{6} a$, which ranges from $\frac{2}{3}$ to $\frac{1}{2}$, and each player in group 2 earns $u_{2}\left(s^{\star}\right)=0$. 


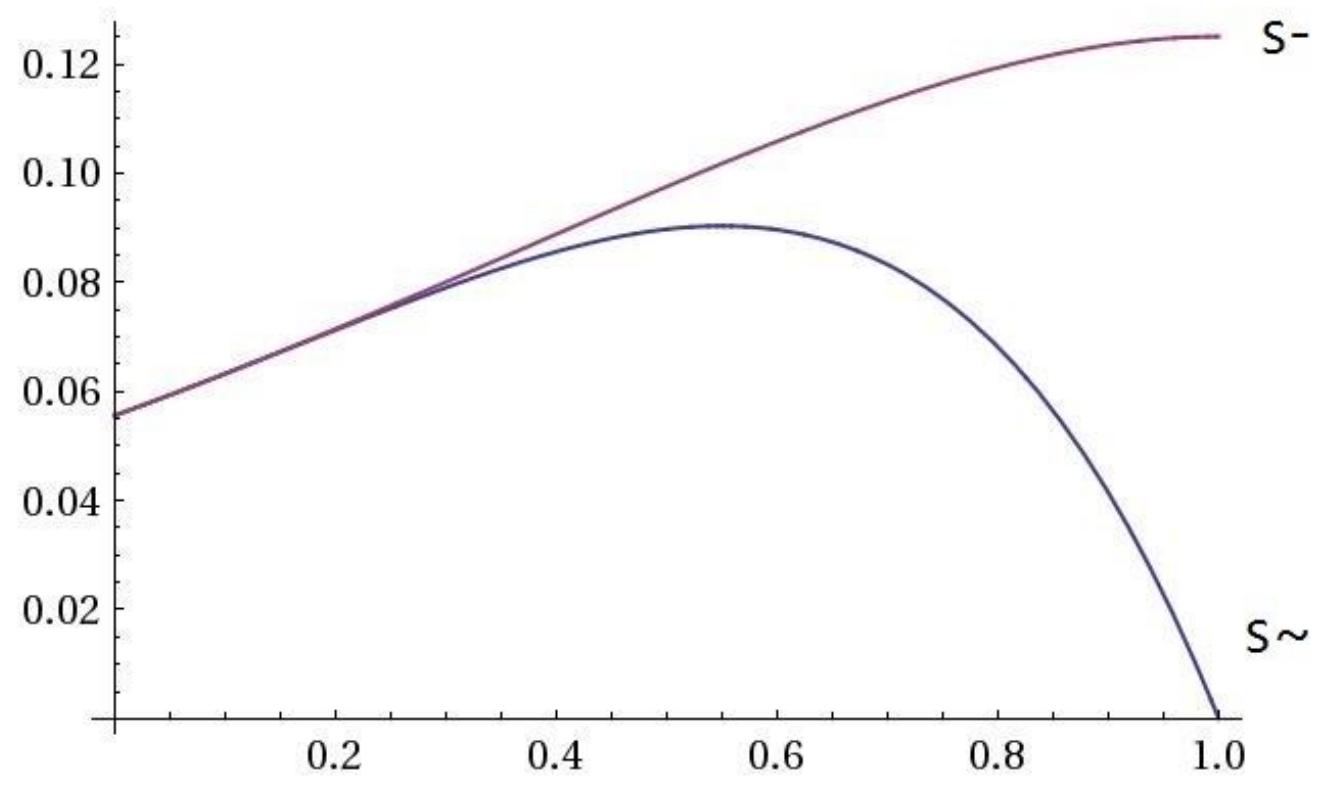

Figure 2. The plot of $\tilde{s}$ and $\bar{s}$ 

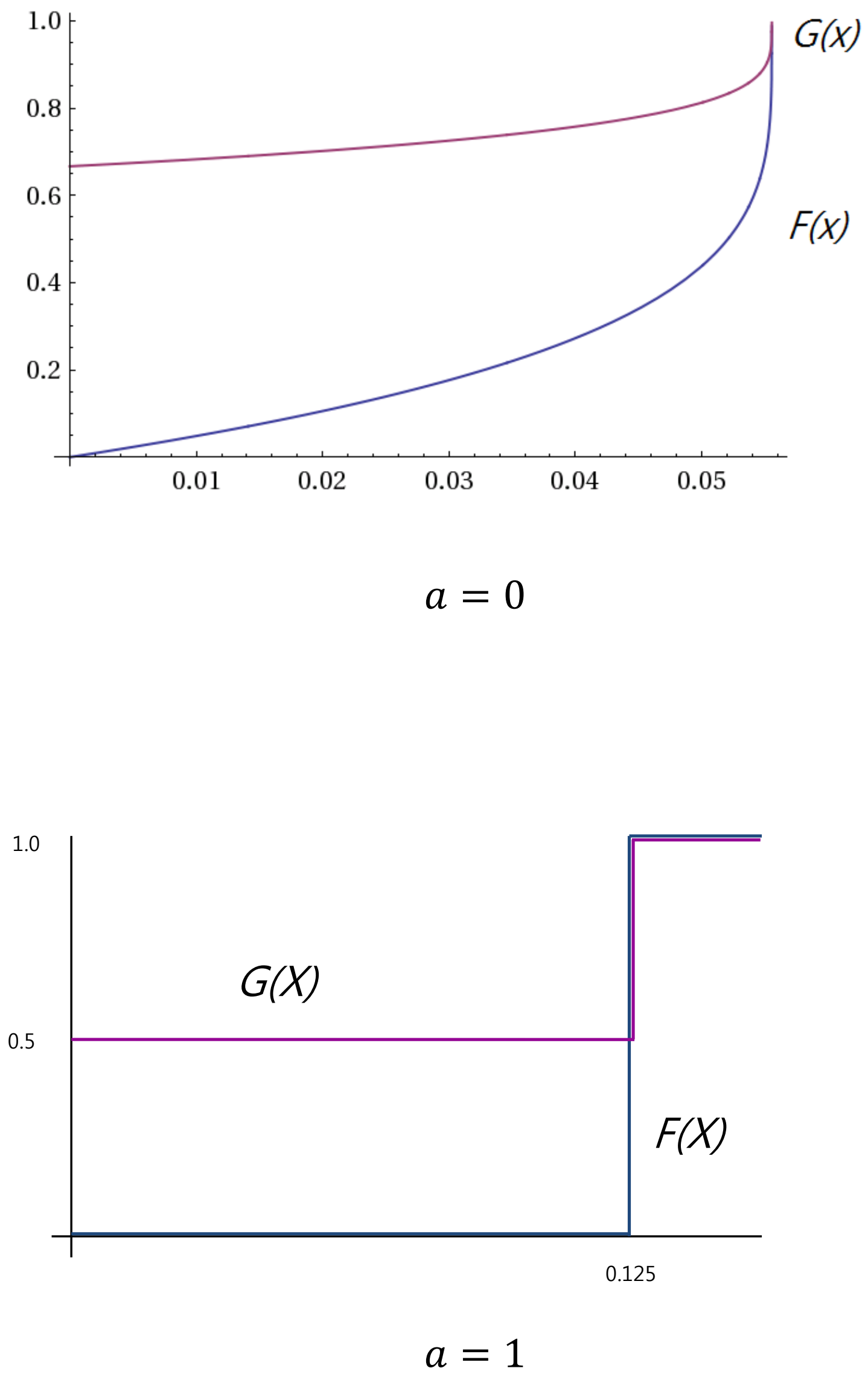

Figure 3. The equilibrium CDF when $a=0$ and $a=1$ 


\section{A general model with $n$ groups}

In the previous section, we addressed the case of two groups where the valuation of the prize was the same for all players within a group. We now extend that model and assume that there are $n>2$ groups, and that individual valuations may differ within as well as across groups. As in Section 2, denote a generic group by $g$. As before, suppose group $g$ has $k_{g} \geq 2$ players. Without loss of generality, $v_{g, 1} \geq v_{g, 2} \geq \cdots \geq v_{g, k_{g}}$ within each group.

Definition 3.1. Given group g's pure strategy $x_{g}$, group g participates if $B\left(x_{g}\right)>0$.

Without loss of generality groups are ordered according to the lowest valuation in the descending order, i.e., $v_{1, k_{1}} \geq v_{2, k_{2}} \geq \cdots \geq v_{g, k_{g}}$. The following result suggests that there exist pure strategy equilibria in which not all groups participate. The number of participating groups depends upon the distribution of valuations.

Let $\mathcal{P}_{n}$ denote the the set of all subsets of $\{1,2, \cdots, n\}$ that contain at least two elements. The following result is a generalization of Theorem 2.8 .

Theorem 3.2. The pure strategy equilibria are as follows. For each subset $J$ of $\mathcal{P}_{n}$,

$$
\begin{gathered}
x_{g, i}=\lambda \quad \text { for all } \quad i \in I_{g}, g \in J, \quad \text { and } \\
x_{g, i}=0 \quad \text { for all } i \in I_{g}, g \notin J,
\end{gathered}
$$

where $0 \leq \lambda \leq \frac{1}{m} \min _{g \in J} v_{g, k_{g}}$, and $m$ is the cardinality of $J$.

To better understand this result, consider the following example.

Example 3.3. Suppose there are 3 groups with valuations $\{1,2\},\{2,3,4\}$, and $\{2,2\}$. Then the following pure strategy equilibria could arise.

(1) No group participates.

(2) All groups participate and all players put the same effort level $\lambda$, where $0 \leq \lambda \leq \frac{1}{3}$.

(3) Only groups 1 and 2 (or groups 1 and 3) participate, and every player in a participating groups expends the same effort $\lambda$ such that $0 \leq \lambda \leq \frac{1}{2}$.

(4) Only groups 2 and 3 participate, and every player in a participating group expends the same effort $\lambda$ such that $0 \leq \lambda \leq 1$. 
One can verify that Lemmata 4.1, 4.2, 4.3 and Corollary 4.4 generalize to the case of asymmetric valuation because the proofs of these results do not rely on the assumption that the valuation is the same within a group. Note that it is hard to solve for equilibria in a more general setting for the following reasons. First, for the common valuation case where $n>2$, one would need to solve a system of $n$ differential equations, containing terms of the form $F_{1}^{\prime} F_{2}^{\prime} \cdots F_{n-1}^{\prime}$. Second, even if $n=2$ but the valuations are asymmetric within a group, different groups may put different atoms at zero, which would significantly complicate the derivation. Combining these two settings together would exacerbate the problem. We conjecture, however, that all symmetric mixed strategy equilibria (if any) must be of the following form.

(1) All players in any of the participating groups randomize continuously and without a gap over some interval $[0, \bar{s}]$, possibly putting mass at zero.

(2) All players in any of the participating groups randomize continuously and without a gap over some interval $[0, \tilde{s}]$ and put mass at some $\bar{s}>\tilde{s}$.

Though the derivation of these equilibria (as well as the payoff configuration) is beyond the scope of our paper and may be an avenue for future research, we only conjecture that high-valuation players put larger mass at $\bar{s}$.

\section{Discussion}

We construct and analyze the 'Max-Min group contest', in which the minimum effort exerted among the group members represents the group effort, and the group with the maximum group effort becomes the winner. Examples of such structure include R\&D competitions, sequential production, supply-chain process, negative product or political campaigns, various sporting events, territorial conflicts. We fully characterize the pure strategy and symmetric mixed strategy equilibria for two groups. We find that there exists a continuum of pure strategy equilibria. In addition, there exist semi-pure strategy equilibria, where all the members of a group play the same pure strategy, whereas all the members of the other group play the same mixed strategy. There are two further types of non-degenerate mixed strategy equilibria, with and without continuous supports. We find that different combinations of the prize valuations and the number of players in each group may result in different types of equilibria with distinct rent dissipation and payoffs. 
For the case of more than two groups, we fully characterize the pure strategy equilibria and point out candidates for mixed strategy equilibria.

Our results are different from those of the weakest-link public goods games, or the weakest-link group contest with the logit-type CSF. While players within each group employ the same strategy, as in those games due to the perfect complementarity, both the variety of equilibria and the corresponding payoffs remain different. The most important part of the difference is the 'group size effect'; in our model the relative group size plays an important role, unlike in the other studies.

The results are also distinguishable from group all-pay auction with other impact functions. Those contests do not possess pure or semi-pure strategy equilibria; while in our model, there is a continuum of pure strategy equilibria. In such equilibria a very wide range (from full to negligible) of rent dissipation is possible. Due to the weakest-link technology, in any equilibrium all players are active provided that the entire group is active. This is in contrast with the free-riding equilibria under the best-shot or perfectly substitute technologies where only a few (possibly one) players are active.

Obtained results such as the existence of a pure or a semi-pure strategy equilibrium, a gap in the equilibrium support, or the group size effect are different from the standard all-pay auction literature. First, it is known that PSNE may exist in the case of incomplete information (Krishna and Morgan, 1997) and for a non-monotonic payoff function (Chowdhury, 2009). Although our model does not satisfy any of those, due to the specific network externalities, a continuum of PSNE exists. It is also uncommon to observe the semi-pure strategy equilibrium, in which some players play a pure strategy whereas some others play a mixed strategy. This type of equilibrium is noted by Krishna (1989) in trade theory and by Clark and Konrad (2007) for multi-battle contest with network effects. We show that such equilibria may even exist in group contests due to the network effects. The effects of network externalities and relative group size result in a jump in equilibrium support which is also not observed in the all-pay auction studies. Finally, unless valuation distribution is changed, the group size does not affect equilibrium strategies and payoffs in standard group contests. We find that in case of the Max-Min group contest, the 'relative valuation' scaled by the group size determines equilibrium effort. This makes parts of the current results comparable to the group contests with private good prizes.

Similar to the minimum-effort coordination game of Van Huyck et al. (1990) and Anderson and Goeree (2001), in which players' effort levels are perfect complements in 
providing public goods, there exist multiple pure and mixed strategy Nash equilibria in our model, since the group impact function for each group depends on the minimum effort level within the group. Hence, the existence of multiple equilibria brings in the relevant and important issues of coordination problems and equilibrium selection principles. Especially, in the common valuation case where all the players in a group have the same valuation of the prize, those issues will be important.

Some standard assumptions employed in our study can be relaxed to explore further avenues. It is possible to extend the structure to cover situations in which the prize is a private good. Some real life situations could be modeled within an incomplete information framework. Finally, the assumption of risk neutrality can be relaxed to incorporate risk aversion. We leave these issues for future research.

\section{Acknowledgements}

We thank Oliver Gürtler, Dan Kovenock, Wolfgang Leininger, Dana Sisak, Seth Streitmatter, Fanis Tsoulouhas, the seminar participants at the City University of London, the University of East Anglia, Indian Statistical Institute, Yonsei University, the participants at the 13th SAET Conference in Paris, and the 2013 Conference on Tournaments, Contests and Relative Performance Evaluation at the University of California, Merced for valuable comments and discussions. We are indebted to Stefano Barbieri and David Malueg for introducing to us an important tool that enabled us to solve a part of the problem. Any remaining errors are our own. 


\section{REFERENCES}

[1] Abernathy, W.J., and Rosenbloom, R. (1968). Parallel and Sequential R\&D Strategies. IEEE Transactions on Engineering Management, 15, 2-10.

[2] Anderson, S.P. and Goeree, J.K. (2001). Minimum-effort coordination games: Stochastic potential and logit equilibrium. Games and Economic Behavior, 34, 177-199.

[3] Arce, M.D.G. (2001). Leadership and the aggregation of international collective action. Oxford Economic Papers, 53, 114-137.

[4] Baik, K.H. (1993). Effort levels in contests: The public-good prize case. Economics Letters, 41, 363-367.

[5] Baik. K.H. (2008). Contests with group-specific public-good prizes. Social Choice and Welfare, 30, 103-117.

[6] Baik, K.H., Kim, I.G., and Na, S. (2001). Bidding for a group-specific public-good prize. Journal of Public Economics, 82, 415-429.

[7] Barbieri, S., Malueg, D.A., and Topolyan, I. (2014). The best-shot all-pay (group) auction with complete information. Economic Theory, 57(3), 603-640.

[8] Baye, M.R., Kovenock, D., and de Vries, C.G. (1996). The all-pay auction with complete information. Economic Theory, 8, 291-305.

[9] Benton, W.C., and Maloni, M. (2005). The influence of power driven buyer/seller relationships on supply chain satisfaction. Journal of Operations Management, 23(1), 1-22.

[10] Bergstrom, T., Blume, L., and Varian, H. (1986). On the private provision of public goods. Journal of Public Economics, 29, 25-49.

[11] Choi, J.P., Chowdhury, S.M., and Kim, J. (2014). Group contests with internal conflict and power asymmetry, University of East Anglia Applied and Financial Economics Working Paper Series, paper No. 25.

[12] Chowdhury, S.M. (2009). The All-pay Auction with non-monotonic Payoff. University of East Anglia ESRC Centre for Competition Policy Working Paper No. 10-6.

[13] Chowdhury, S.M., and Gürtler, O. (2013). Sabotage in Contests: A Survey. Available at SSRN 2338077.

[14] Chowdhury, S.M., Lee, D., and Sheremeta, R. (2013). Top guns may not fire: best-shot group contests with group-specific public good prizes. Journal of Economic Behavior and Organization, 92, 94-103.

[15] Chowdhury, S.M. and Topolyan, I. (2013). The attack-and-defense group contests. Working paper, available at http://ideas.repec.org/s/uea/aepppr.html.

[16] Clark, D.J. and Konrad, K.A. (2007). Asymmetric Conflict: Weakest Link against Best Shot. Journal of Conflict Resolution, 51, 457-469. 
[17] Coddington, E.A. and Levinson, N. (1955). Theory of ordinary differential equations. New York: McGraw Hill.

[18] Conybeare, J.A.C., Murdoch, J.C., and Sandler., T. (1994). Alternative Collective-Goods Models of Military Coalitions: Theory and Empirics. Economic Inquiry, 32, 525-542.

[19] Cornes, R. and Hartley, R. (2007). Weak links, good shots and other public good game: Building on BBV. Journal of Public Economics, 91, 1684-1707.

[20] Dijkstra, B.R. (1998). Cooperation by way of support in a rent-seeking contest for a public good. European Journal of Political Economy, 14, 703-725.

[21] Hansen, M. T., and Birkinshaw, J. (2007). The innovation value chain. Harvard business review, 85(6), 121.

[22] Harrison, G.W., and Hirshleifer, J. (1989). An experimental evaluation of weakest link/best shot models of public goods. Journal of Political Economy, 97, 201-225.

[23] Hillman, A.L., and Riley, J.G. (1989). Politically contestable rents and transfers. Economics and Politics, 1, 17-39.

[24] Hirshleifer, J. (1983). From weakest-link to best-shot: The voluntary provision of public goods. Public Choice, 41, 371-386.

[25] Kaplan, T. R., Luski, I., and Wettstein, D. (2003). Innovative activity and sunk cost. International Journal of Industrial Organization, 21(8), 1111-1133.

[26] Katz, E., Nitzan, S., and Rosenberg, J. (1990). Rent-seeking for pure public goods. Public Choice, 65, 49-60.

[27] Kolmar, M. and Rommeswinkel, H. (2013). Group Contests with Group-specific Public Good and Complementarities in Efforts. Journal of Economic Behavior and Organization, 89, 9-22.

[28] Konrad, K. (2009). Strategy and Dynamics in Contests. New York: Oxford University Press.

[29] Kovenock, D. and Roberson, B. (2010). The Optimal Defense of Networks of Targets. Purdue University Working Paper No. 1251.

[30] Kremer, M. (1993). The O-Ring theory of economic development. Quarterly Journal of Economics, 108, 551-575.

[31] Krishna, K. (1989). Trade Restrictions as Facilitating Practices. Journal of International Economics, 26, 251-270.

[32] Krishna, V. and Morgan, J. (1997). An Analysis of the War of Attrition and the All-Pay Auction. Journal of Economic Theory, 72, 343-362.

[33] Lee, D. (2012). Weakest-link contests with group-specific public good prizes. European Journal of Political Economy, 28, 238-248.

[34] Lei, V., Tucker, S., and Vesely, F. (2007). Foreign aid and weakest-link international public goods: An experimental study. European Economic Review, 51, 599-623.

[35] Münster, J. (2009). Group contest success functions. Economic Theory, 41, 345-357. 
[36] Riaz, K., Shogren, J.F., Johnson, S.R. (1995). A general model of rent-seeking for public goods. Public Choice, 82, 243-259.

[37] Nelson, R.R. (1961). Uncertainty, Learning, and the Economics of Parallel Research and Development efforts. Review of Economics and Statistics, 43, 351-364.

[38] Sandler, T. and Vicary, S. (2001). Weakest-link public goods: Giving in-kind or transferring money in a sequential game. Economics Letters, 74, 71-75.

[39] Skaperdas, S. and Grofman, B. (1995). Modeling negative campaigning. American Political Science Review, 89, 49-61.

[40] Slone, R.E., Mentzer, J.T., and Dittmann, J.P. (2007). Are you the weakest link in your company's supply chain?. Harvard Business Review, 85(9), 116.

[41] Topolyan, I. (2014). Rent-seeking for a public good with additive contributions. Social Choice and Welfare, 42(2), 465-476.

[42] Tullock, G. (1980). Efficient Rent Seeking. In James M. Buchanan, Robert D. Tollison, Gordon Tullock, (Eds.), Toward a theory of the rent-seeking society. College Station, TX: Texas A\&M University Press, 97-112.

[43] Ursprung, H.W. (1990). Public goods, rent dissipation, and candidate competition. Economics and Politics, 2, 115-132.

[44] Van Huyck, J.B., Battalio, R.C., and Beil, R.O. (1990). Tacit coordination games, strategic uncertainty, and coordination failure. American Economic Review, 80, 234-248.

[45] Varian, H.A. (2004). System Reliability and Free Riding, Economics of Information Security. Advances in Information Security, 12, 1-15.

[46] Vicary, S. (1990). Transfer and the weakest-link: An extension of Hirshleifer's analysis. Journal of Public Economics, 43, 375-394.

[47] Vicary, S. and Sandler, T. (2002). Weakest-link public goods: Giving in-kind or transferring money. European Economic Review, 46, 1501-1520.

[48] Wärneryd, K. (1998). Distributional conflict and jurisdictional organization. Journal of Public Economics, 69, $435-450$. 


\section{Appendix: Proofs of the results}

Lemma 4.1. Let $s^{\star}$ be a symmetric mixed strategy equilibrium, then the following statements are true.

(1) $\bar{s}_{g, i}^{\star}=\bar{s}_{g}^{\star}$ for all $i \in I_{g}$.

(2) $S_{1+}^{\star}=S_{2+}^{\star}$, particularly $\underline{s}_{1+}^{\star}=\underline{s}_{2+}^{\star}=\underline{s}_{+}^{\star}$.

(3) If $S_{1}^{\star}=S_{2}^{\star}$, then $\underline{s}^{\star}=0$, where $\underline{s}^{\star}$ is the common lower bound of the supports.

(4) At most one group puts mass at zero.

Proof. (1) Suppose without loss of generality that $\bar{s}_{g, i}^{\star}>\bar{s}_{g, j}^{\star}$, then player $j$ is better-off shifting mass from $\left(\bar{s}_{g, i}^{\star}, \bar{s}_{g, j}^{\star}\right]$ to $\bar{s}_{g, i}^{\star}$, leading to a contradiction.

(2) Suppose $S_{1+}^{\star} \neq S_{2+}^{\star}$. Without loss of generality let $x$ be such that $x \in S_{1+}^{\star}$ but $x \notin S_{2+}^{\star}$. Consider the following cases.

(a) If $x>\bar{s}_{2+}^{\star}$, then any player in group 1 is better-off switching from $x$ to $x-\epsilon$ for some small $\epsilon>0$, which is a contradiction.

(b) If $x<\underline{s}_{2+}^{\star}$, then any player in group 1 is better-off deviating from $x$ to 0 (observe that $x>0$ since $x \in S_{1+}^{\star}$ ).

(c) If $\underline{s}_{2}^{\star}<x<\bar{s}_{2}^{\star}$ (in which case the support of group 2 is not connected), then any player in group 1 would deviate from $x$ to $x-\epsilon$ for some small $\epsilon>0$.

Therefore $S_{1+}^{\star}=S_{2+}^{\star}$, which implies $\underline{s}_{1+}^{\star}=\underline{s}_{2+}^{\star}=\underline{s}_{+}^{\star}$.

(3) Suppose that $S_{1}=S_{2}$ and the common lower bound is $\underline{s}^{\star}>0$. We claim that every group must put an atom at $\underline{s}^{\star}$. Suppose not, say group 1 does not put an atom at $\underline{s}^{\star}$, then the payoff of any player $i$ in group 2 from playing $\underline{s}^{\star}$ is

$$
u_{2, i}\left(\underline{s}^{\star}, s_{-i}^{\star}\right)=-\underline{s}^{\star}<0 .
$$

Hence player $i$ is better-off deviating to 0 , which establishes the claim. But if every group puts some mass at $\underline{s}^{\star}$, then any player could increase her payoff by deviating from $\underline{s}^{\star}$ to $\underline{s}^{\star}+\epsilon$ for some small $\epsilon>0$, which is a contradiction.

(4) Suppose not, then any player would shift mass from zero to a very small positive effort.

Lemma 4.2. Let $s^{\star}$ be a symmetric mixed strategy equilibrium, then no player puts mass at $x$ for any $x \in\left[\underline{s}_{+}^{\star}, \bar{s}^{\star}\right) \cap \mathbb{R}_{++}$. 
Proof. First, let us show that no player puts mass in $\left(\underline{s}_{+}^{\star}, \bar{s}^{\star}\right)$. Suppose without loss of generality group 1 puts mass at $a$ such that $\underline{s}_{+}^{\star}<a<\bar{s}^{\star}$. It is easy to see that $F_{1}$ has a jump discontinuity at $a$. Then any player in group 2 would shift mass from $(a-\epsilon, a]$ to $a+\delta$ for some small $\epsilon, \delta>0$. If no member of group 2 puts mass in $(a-\epsilon, a]$, then it is not optimal for group 1 to put mass at $a$, which establishes a contradiction.

It remains to show that if $\underline{s}_{+}^{\star}>0$, then no player puts mass at $\underline{s}_{+}^{\star}$. Assume $\underline{s}_{+}^{\star}>0$, and observe, following the argument of Lemma4.1(3), that at most one group puts mass at $\underline{s}_{+}^{\star}$. Without loss of generality, suppose group 1 puts mass at $\underline{s}_{+}^{\star}$ and group 2 does not. But then group 1 is better-off shifting mass from $\underline{s}_{+}^{\star}$ to some very small positive effort since no group puts mass in $\left(0, \underline{s}_{+}^{\star}\right)$, contradicting our assumption.

Proof of Theorem 2.10. Let $s^{\star}$ be an equilibrium which is symmetric and semi-pure. By Lemma 4.1(2) we have $S_{1+}^{\star}=S_{2+}^{\star}$. By Lemma 4.2 no player puts mass above zero. Notice that it is not possible for both groups to put mass at zero, for otherwise any player of the other group could increase her payoff by deviating from zero to some small $\epsilon>0$. Therefore the only possibility is for every player in one group to always contribute some $x>0$ and for every player in the other group (group $g$ ) to randomize between zero and $x$. Let every player in group $g$ put mass $1-a$ at $x$. Since every player in group $g$ earns zero payoff and is indifferent between his pure strategies of zero and $x$, it must be that

$$
x=\frac{v_{g}}{2}(1-a)^{k_{g}-1} .
$$

Clearly, no player $j$ in group $(-g)$ wants to deviate from $x$ provided that

$$
u_{-g, j}\left(s^{\star}\right)=v_{-g}\left(1-\frac{1}{2}(1-a)^{k_{g}}\right)-x \geq 0,
$$

which is satisfied if and only if

$$
\frac{2-(1-a)^{k_{g}}}{(1-a)^{k_{g}-1}} \geq \frac{v_{g}}{v_{-g}}
$$

Condition 4.1 holds if and only if $v_{g} \leq v_{-g}$, i.e., $v_{g}=v_{2}$ and $v_{-g}=v_{1}$, in which case it holds for all $0 \leq 1-a \leq 1$. This completes the proof. 
Lemma 4.3. Let $s^{\star}$ be a symmetric mixed strategy equilibrium for which there exists $0<z<\bar{s}^{\star}$ such that $x \in S_{1} \cap S_{2}$ for all $z \leq x \leq \bar{s}^{\star}$. Then no player puts mass at $\bar{s}^{\star}$ (i.e., it is not the case that all players of some group put an atom at $\left.\bar{s}^{\star}\right)$.

Proof. Suppose not, and without loss of generality group 1 puts an atom at $\bar{s}^{\star}$. Consider two possible cases.

(1) Group 2 also puts a positive mass at $\bar{s}^{\star}$. Then any player $i$ in group 1 is betteroff increasing her effort from $\bar{s}^{\star}-\epsilon$ to $\bar{s}^{\star}$ for a sufficiently small $\epsilon>0$ for the following reason. If player $i$ expends effort level $\bar{s}^{\star}$, then group 1 ties with group 2 with a positive probability (in the event that the effort level of both groups is $\bar{s}^{\star}$ ). However if player $i$ expends effort $\bar{s}^{\star}-\epsilon$, then group 1's effort is always less than $\bar{s}^{\star}$ due to the weakest-link effort technology, and group 1 does not get a chance to tie with group 2 at $\bar{s}^{\star}$, resulting in a loss of payoffs for all players in group 1. For a sufficiently small $\epsilon$ such loss exceeds the economy on the effort cost for player $i$, which is equal to $\epsilon$. This implies that the support $S_{1, i}^{\star}$ is disconnected, contradicting our initial assumption.

(2) Group 2 does not put a positive mass at $\bar{s}^{\star}$. Again, we claim that any player $i$ in group 1 is better-off increasing her effort from $\bar{s}^{\star}-\epsilon$ to $\bar{s}^{\star}$ for a sufficiently small $\epsilon>0$. If player $i$ chooses $\bar{s}^{\star}$, then with a positive probability group 1's effort exceeds that of group 2 (which happens when group 1's effort is $\bar{s}^{\star}$. If player $i$ expends effort level $\bar{s}^{\star}-\epsilon$, then group 1's effort is always less than $\bar{s}^{\star}$, which results in a payoff loss for player $i$. Consequently, the support $S_{1, i}^{\star}$ is disconnected, contradicting our initial assumption.

Corollary 4.4. Let $s^{\star}$ be a symmetric mixed strategy equilibrium which is not semi-pure, then the equilibrium group support $S_{g}^{\star}$ has uncountably many elements for each $g=1,2$.

Proof. Immediate consequence of Lemmata 4.1(2), 4.1(3), and 4.2.

Proof of Theorem 2.11. Suppose $s^{\star}$ is an equilibrium such that the support of each player's strategy is the interval $\left[0, \bar{s}^{\star}\right]$. Recall that the effort distribution of group $g$, given 
the individual distribution functions $F_{g, i}$ 's of its players, is given by

$$
F_{g}(x)=1-\prod_{i \in I_{g}}\left(1-F_{g, i}(x)\right) \text { for each } 0 \leq x \leq \bar{s}^{\star} .
$$

Equation 2.4 implies that the payoff of player $i$ in group 1 from expending an effort $x \in\left(0, \bar{s}^{\star}\right]$ is

$$
u_{1, i}(x)=v_{1}\left[\int_{0}^{x} F_{2}(z) d F_{1,-i}(z)+F_{2}(x)\left(1-F_{1,-i}(x)\right)\right]-x
$$

Since $F_{2}$ and $F_{1,-i}$ are continuous on $\left(0, \bar{s}^{\star}\right]$ (due to the continuity of each player's strategy by Lemmata 4.2 and 4.3), $u_{1, i}(x)$ is continuous on the same interval. Because player $i$ is randomizing over $\left(0, \bar{s}^{\star}\right]$ without a gap, $u_{1, i}$ is constant on a dense subset of $\left(0, \bar{s}^{\star}\right]$. Combined with the fact that $u_{1, i}$ is continuous, we conclude that $u_{1, i}$ is constant on that interval. Therefore, $u_{1, i}$ is continuously differentiable on $\left(0, \bar{s}^{\star}\right)$, and the derivative is equal to zero. ${ }^{9}$ Thus,

$$
\begin{aligned}
u_{1, i}^{\prime}(x) & =v_{1}\left[F_{2}(x) F_{1,-i}^{\prime}(x)+F_{2}^{\prime}(x)-\left(F_{2}^{\prime}(x) F_{1,-i}(x)+F_{2} F_{1,-i}^{\prime}(x)\right)\right]-1 \\
& =v_{1} F_{2}^{\prime}(x)\left[1-F_{1,-i}(x)\right]-1 \\
& =0
\end{aligned}
$$

Denote the cdf of a generic player in group 1 by $F$, and the cdf of a generic player in group 2 by $G$, then 4.2 implies that $F_{2}(x)=1-(1-G(x))^{k_{2}}$ and $F_{1,-i}(x)=1-(1-F(x))^{k_{1}-1}$. Therefore

$$
v_{1} k_{2}(1-G(x))^{k_{2}-1} G^{\prime}(x)(1-F(x))^{k_{1}-1}=1
$$

Now fix player $j$ in group 2, and follow the same lines to conclude that

$$
v_{2} k_{1}(1-G(x))^{k_{2}-1} F^{\prime}(x)(1-F(x))^{k_{1}-1}=1
$$

Equations 4.4 and 4.5 imply that $\frac{v_{2} k_{1}}{v_{1} k_{2}} F^{\prime}(x)=G^{\prime}(x)$ for all $x \in\left(0, \bar{s}^{\star}\right)$.

1. Assume $\frac{v_{2} k_{1}}{v_{1} k_{2}} \leq 1$. Notice that

$$
1-G(x)=\int_{x}^{\bar{s}^{\star}} G^{\prime}(z) d z=\frac{k_{1} v_{2}}{k_{2} v_{1}} \int_{x}^{\bar{s}^{\star}} F^{\prime}(z) d z=\frac{k_{1} v_{2}}{k_{2} v_{1}}(1-F(x))
$$

Substituting this into 4.4 yields

$$
k_{2} v_{1}\left(\frac{k_{1} v_{2}}{k_{2} v_{1}}\right)^{k_{2}}(1-F(x))^{k_{1}+k_{2}-2} F^{\prime}(x)=1
$$

\footnotetext{
${ }^{9}$ We are indebted to David A. Malueg and Stefano Barbieri for introducing this technique to us.
} 
The above differential equation determines $F$. To calculate $F$, integrate both sides of 4.20 .

$$
k_{2} v_{1}\left(\frac{k_{1} v_{2}}{k_{2} v_{1}}\right)^{k_{2}} \frac{1}{k_{1}+k_{2}-1}(1-F(x))^{k_{1}+k_{2}-1}=C-x
$$

where $C$ is a constant of integration. Therefore, $F$ is of the form:

$$
F(x)=1-\left(\frac{c_{1}-x}{c_{2}}\right)^{\frac{1}{k_{1}+k_{2}-1}},
$$

where $c_{1}$ and $c_{2}$ are some constants. Observing that $F(0)=0$ and $F\left(\bar{s}^{\star}\right)=1$, we conclude that $c_{1}=c_{2}=\bar{s}^{\star}$, therefore

$$
\begin{gathered}
F(x)=1-\left(1-\frac{x}{\bar{s}^{\star}}\right)^{\frac{1}{k_{1}+k_{2}-1}}, \text { and } \\
G(x)=\left(1-\frac{k_{1} v_{2}}{k_{2} v_{1}}\right)+\frac{k_{1} v_{2}}{k_{2} v_{1}} F(x)=1-\frac{k_{1} v_{2}}{k_{2} v_{1}}\left(1-\frac{x}{\bar{s}^{\star}}\right)^{\frac{1}{k_{1}+k_{2}-1}}
\end{gathered}
$$

Notice that each player in group 2 places mass $\left[1-\frac{k_{1} v_{2}}{k_{2} v_{1}}\right]$ at zero (hence group 2 places mass $\left[1-\left(\frac{k_{1} v_{2}}{k_{2} v_{1}}\right)^{k_{2}}\right]$ at zero), while group 1 randomizes continuously over $\left[0, \bar{s}^{\star}\right]$. Therefore the expected payoff of any player in group 1 is $v_{1}\left[1-\left(\frac{k_{1} v_{2}}{k_{2} v_{1}}\right)^{k_{2}}\right]$, while any player in group 2 earns zero expected payoff. To pin down $\bar{s}^{\star}$, observe that by differentiating 4.9 we obtain

$$
F^{\prime}(x)=\frac{1}{\bar{s}^{\star}\left(k_{1}+k_{2}-1\right)}\left(1-\frac{x}{\bar{s}^{\star}}\right)^{2-k_{1}-k_{2}}=\frac{1}{\bar{s}^{\star}\left(k_{1}+k_{2}-1\right)}(1-F(x))^{2-k_{1}-k_{2}}
$$

Express $F^{\prime}(x)$ from 4.20 and equate it to the right hand side of 4.11 , which yields

$$
\bar{s}^{\star}=\left(\frac{k_{1} v_{2}}{k_{2} v_{1}}\right)^{k_{2}} \cdot \frac{k_{2} v_{1}}{k_{1}+k_{2}-1}
$$

Equations 4.9, 4.10, and 4.12 characterize a mixed strategy equilibrium under the condition $\frac{v_{2} k_{1}}{v_{1} k_{2}} \leq 1$, which is unique in the subclass of strategy profiles where players randomize over an interval without a gap.

2. Assume $\frac{v_{2} k_{1}}{v_{1} k_{2}}>1$. Follow the lines of the previous case to conclude that $\bar{s}^{\star}=$ $\left(\frac{k_{2} v_{1}}{k_{1} v_{2}}\right)^{k_{1}} \cdot \frac{k_{1} v_{2}}{k_{1}+k_{2}-1}, G(x)=1-\left(1-\frac{x}{\bar{S}^{\star}}\right)^{\frac{1}{k_{1}+k_{2}-1}}$, and $F(x)=\left(1-\frac{k_{2} v_{1}}{k_{1} v_{2}}\right)+\frac{k_{2} v_{1}}{k_{1} v_{2}} G(x)$. 
Proof of Theorem 2.14. Suppose $s^{\star}$ is a symmetric equilibrium such that the support of each player is the union $\left[0, \tilde{s}^{\star}\right] \cup \bar{s}^{\star}$. Let $a_{g}$ be the mass that each player in group $g$ puts at $\bar{s}^{\star}$. As any player $i$ is indifferent between $\tilde{s}^{\star}$ and $\bar{s}^{\star}$, for $g=1,2$ we have

$$
u_{g, i}\left(\bar{s}_{g, i}^{\star}, s_{-i}^{g \star}, s_{-g}^{\star}\right)-u_{g, i}\left(\hat{s}_{g, i}^{\star}, s_{-i}^{g \star}, s_{-g}^{\star}\right)=\frac{v_{g}}{2}\left(a_{-g}\right)^{k_{-g}}\left(a_{g}\right)^{k_{g}-1}-\left(\bar{s}^{\star}-\hat{s}^{\star}\right)=0,
$$

Consequently,

which yields

$$
\frac{a_{1}}{a_{2}}=\frac{v_{1}}{v_{2}}
$$

$$
\bar{s}^{\star}-\hat{s}^{\star}=\frac{v_{1}}{2}\left(\frac{v_{2}}{v_{1}}\right)^{k_{2}} a_{1}^{k_{1}+k_{2}-1} .
$$

Clearly, $0<a_{1}<1$ and $0<a_{2}<1$ must be satisfied. Observe that player $i$ is indifferent between expending effort levels $\hat{s}^{\star}$ and $z$ for any $0<z<\hat{s}^{\star}$. For each $x \in\left[0, \hat{s}^{\star}\right]$ the expected payoff of player $i$ in group 1 is

$$
u_{1, i}(x)=v_{1}\left[\int_{0}^{x} F_{2}(z) d F_{1,-i}(z)+F_{2}(x)\left(1-F_{1,-i}(x)\right)\right]-x .
$$

Follow the lines of Theorem 2.11 to conclude that Equations 4.4 and 4.5 hold for all $0 \leq x \leq \hat{s}^{\star}$, thus $\frac{k_{1} v_{2}}{k_{2} v_{1}} F^{\prime}=G^{\prime}$. Note that for $0 \leq x \leq \hat{s}^{\star}$ we have

$$
1-a_{1} \cdot \frac{v_{2}}{v_{1}}-G(x)=\int_{x}^{\hat{s}^{\star}} G^{\prime}(z) d z=\frac{k_{1} v_{2}}{k_{2} v_{1}} \int_{x}^{\hat{s}^{\star}} F^{\prime}(z) d z=\frac{k_{1} v_{2}}{k_{2} v_{1}}\left(1-a_{1}-F(x)\right) .
$$

We thus have the following candidates for equilibrium cdfs on $\left[0, \hat{s}^{\star}\right]$.

(1) Type I equilibrium:

$$
G(x)=\left[1-a_{1} \frac{v_{2}}{v_{1}}-\frac{k_{1} v_{2}}{k_{2} v_{1}}\left(1-a_{1}\right)\right]+\frac{k_{1} v_{2}}{k_{2} v_{1}} F(x),
$$

where $F$ is strictly increasing and $F(0)=0 .{ }^{10}$

(2) Type II equilibrium:

$$
F(x)=\left[1-a_{1}-\frac{k_{2} v_{1}}{k_{1} v_{2}}+\frac{k_{2}}{k_{1}} a_{1}\right]+\frac{k_{2} v_{1}}{k_{1} v_{2}} G(x),
$$

such that $G$ is strictly increasing and $G(0)=0$.

Consider three possible cases:

\footnotetext{
$\overline{10}$ This is because $F$ and $G$ cannot both have atoms at zero, for otherwise any player would deviate from zero to a very small positive effort. Note that this is not always the case in an individual all-pay auction. We proved earlier that every player within a group plays the same strategy, thus if $F$ had an atom at zero, then the effort distribution of the entire group (group 1) would have an atom at zero.
} 
(1) $k_{1}=k_{2}$; it should be evident that type II equilibrium does not exist because $1-a_{1}-\frac{k_{2} v_{1}}{k_{1} v_{2}}+\frac{k_{2}}{k_{1}} a_{1}=1-\frac{v_{1}}{v_{2}}<0$. Let us investigate type I equilibria. When $k_{1}=k_{2}$, Equation 4.18 implies that $G$ puts mass $1-\frac{v_{2}}{v_{1}}$ at zero for any $0<a_{1}<1$. The case $a_{1}=0$ is a degenerate case where all players randomize without a gap; it is taken care of in Theorem 2.11. When $a_{1}=1$ the equilibrium degenerates to the semi-pure equilibrium of Theorem 2.10.

(2) $k_{1}>k_{2}$, then type I equilibrium exists only if $\max \left\{0, \frac{k_{1} v_{2}-k_{2} v_{1}}{v_{2}\left(k_{1}-k_{2}\right)}\right\} \leq a_{1}<1,{ }^{11}$ and type II equilibrium exists only if $\frac{k_{1} v_{2}}{k_{2} v_{1}}>1$ and $0<a_{1} \leq \frac{k_{1} v_{2}-k_{2} v_{1}}{v_{2}\left(k_{1}-k_{2}\right)}$ (these conditions are implied by the fact that the atoms at zero should be non-negative). Note that when $a_{1}=\frac{k_{1} v_{2}-k_{2} v_{1}}{v_{2}\left(k_{1}-k_{2}\right)}$, neither $F$ nor $G$ put mass at zero, so type I and type II equilibria blend into a single equilibrium with $F(0)=G(0)=0$ and $G(x)=\frac{k_{1} v_{2}}{k_{2} v_{1}} F(x)$ for all $0 \leq x \leq \hat{s}^{\star}$.

(3) $k_{1}<k_{2}$, then type I equilibrium exists only if $0<a_{1} \leq \frac{k_{1} v_{2}-k_{2} v_{1}}{v_{2}\left(k_{1}-k_{2}\right)}$, while type II equilibrium fails to exist because under the condition $v_{1}>v_{2}$ the mass placed by $F$ at zero cannot be at most one. Note that since $\frac{k_{1} v_{2}-k_{2} v_{1}}{v_{2}\left(k_{1}-k_{2}\right)}>1$, the necessary condition for type I equilibrium becomes $0<a_{1}<1$.

To verify that the above candidates are equilibria and solve for $F$ and $G$ one needs to plug Equations 4.18 and 4.19 into 4.4. This yields the initial-value problems.

$$
\begin{aligned}
& k_{1} v_{2}\left[\frac{k_{1} v_{2}}{k_{2} v_{1}}-a_{1} \frac{v_{2}}{v_{1}}\left(\frac{k_{1}}{k_{2}}-1\right)-\frac{k_{1} v_{2}}{k_{2} v_{1}} F(x)\right]^{k_{2}-1}[1-F(x)]^{k_{1}-1} F^{\prime}(x)=1, \quad F(0)=0 \\
& k_{2} v_{1}\left[a_{1}+\frac{k_{2} v_{1}}{k_{1} v_{2}}-\frac{k_{2}}{k_{1}} a_{1}-\frac{k_{2} v_{1}}{k_{1} v_{2}} G(x)\right]^{k_{2}-1}[1-G(x)]^{k_{2}-1} G^{\prime}(x)=1, G(0)=0
\end{aligned}
$$

To solve it, we need to employ the basic techniques of the theory of the first-order ordinary differential equations. There is no closed-form solution to the initial-value problems 4.20 and 4.21, however each possesses a unique solution by Picard-Lindelöf theorem (Coddington and Levinson, 1955, Theorem 3.1, p.12). Indeed, $F^{\prime}$ is differentiable in $F$ and continuous in $x$, when one expresses $F^{\prime}$ as

$$
F^{\prime}=\frac{1}{k_{1} v_{2}}\left[\frac{k_{1} v_{2}}{k_{2} v_{1}}-a_{1} \frac{v_{2}}{v_{1}}\left(\frac{k_{1}}{k_{2}}-1\right)-\frac{k_{1} v_{2}}{k_{2} v_{1}} F(x)\right]^{1-k_{2}}[1-F(x)]^{1-k_{1}} .
$$

\footnotetext{
${ }^{11}$ It is easy to verify that $\frac{k_{1} v_{2}-k_{2} v_{1}}{v_{2}\left(k_{1}-k_{2}\right)}<1$ since $v_{1}>v_{2}$.
} 
Similarly, $G^{\prime}$ is differentiable in $G$ and continuous in $x$. Thus, the strategy profiles listed in the above three cases are indeed equilibria. To solve for a type I equilibrium, one needs to solve the initial-value problem 4.20 , then find $\tilde{s}$ as the solution to $F(\hat{s})=1-a_{1}$, and finally pin down $\bar{s}$ from Equation 4.15. Type II equilibrium is solved similarly using 4.21.

Proof of Theorem 3.2. Fix a subset $J$ of $\{1,2, \cdots, n\}$ having $m \geq 2$ elements, and $\lambda \in\left[0, \frac{1}{m} \min _{g \in J} v_{g, k_{g}}\right]$. Intuitively, $J$ is the list of participating groups. Consider a strategy profile $s$ such that each player of any group in $J$ contributes $\lambda$, and the rest of the players do not participate. It is clear that no player in group $\ell \notin J$ wants to deviate by contributing a positive amount, because group $k$ 's effort would still be zero, and the deviator would get a negative payoff. It remains to show that no player of any group in $J$ wants to deviate. Suppose player $i$ of some group $k \in J$ deviates to some $s^{\prime}>\lambda$. It is easy to see that the winning probability of group $k$ is the same, hence $u_{k, i}\left(s^{\prime}, s_{-i}\right)<u_{k, i}(s)$, which is a contradiction. Suppose player $i$ deviates to some $\hat{s}<\lambda$, then group $k$ loses with certainty, hence $u_{k, i}\left(\hat{s}, s_{-i}\right)<u_{k, i}(s)$, leading to a contradiction. This completes the proof. 